\title{
Corneal Nerves Alteration Associated with Corneal Complications after Pars Plana Vitrectomy
}

\author{
Tiezhu Lin ${ }^{1}$, Hong Ye ${ }^{1,2}$, Emmanuel Eric Pazo ${ }^{1}$, Guangzheng Dai ${ }^{1}$, Yang Xia ${ }^{1}$, Wei $\mathrm{He}^{1}$ \\ ${ }^{1}$ Department of Vitreous and Retina, He Eye Specialist Hospital, Shenyang, China \\ ${ }^{2}$ Department of Vitreous and Retina, Bright Eye Hospital, Hefei, China
}

Purpose: To evaluate the effect of corneal nerves assessment on predicting corneal complications following pars plana vitrectomy (PPV).

Methods: In this prospective single-center cohort study, 94 patients (94 eyes) received PPV, and were divided into postoperative groups with and without corneal complications. All eyes had corneal nerve fiber length (CNFL), corneal nerve fiber density, and branch density of corneal nerve fibers assessed and calculated with Image J preoperatively. Multivariate logistic regression analysis was used to identify corneal nerve fiber parameters that correlated to post-operative corneal complications. Receiver operator characteristic curve analysis was performed to identify the optimal cut-off point of the corneal fibers' parameters for predicting corneal complications after PPV.

Results: Eleven eyes (11.70\%) developed corneal complications at 1 week after PPV. There was significant difference between CNFL (19.44 \pm 6.88 vs. $26.84 \pm 7.53, p=0.003)$, corneal nerve fiber density $(28.82 \pm 9.91$ vs. $37.10 \pm 10.16, p=0.013)$ and branch density of corneal nerve fibers $(55.84 \pm 21.08$ vs. $82.04 \pm 31.89, p=0.01)$ in two groups, respectively. Receiver operator characteristic analysis showed that the optimal cutoff value of CNFL to predict corneal complications following PPV was $<26.495 \mathrm{~mm} / \mathrm{mm}^{2}$.

Conclusions: The decrease of CNFL may predict corneal complications following PPV. Regular preoperative corneal confocal microscopy test in PPV patients could be considered.

Key Words: Microscope, Nerve fibers, Postoperative complications, Vitrectomy

Corneal complications such as corneal epithelial defects, corneal edema, or superficial punctate keratopathy are common after pars plana vitrectomy (PPV), which may ad-

Received: March 31, 2021 Final revision: May 5, 2021

Accepted: May 17, 2021

Corresponding Author: Tiezhu Lin, MD. Department of Retinal and Vitreous Disease, He Eye Specialist Hospital, No. 128 Huanghe Street, Yuhong District, Shenyang, Liaoning Province 110000, China. Tel: 86136-5403-2699, Fax: 86-024-2397-4661, E-mail: lintiezhu@hsyk.com.cn vance to ocular discomfort, poor vision, infection, scaring of the cornea, and corneal melting [1,2]. Though previous studies found that risk factors for poor corneal status after PPV are diabetes mellitus (DM), longer operative time, rhegmatogenous retinal detachment, intravitreal tamponade with perfluoropropane, and assistance of a first-year resident were risk factors for poor corneal status after PPV [1,3], the biomarkers have not been found. Corneal confocal microscopy (CCM) assessment is a rapid and reproducible technique that quantifies corneal nerve morphology, and 
the intra class correlation coefficient values were $0.94,0.95$, and 0.96 for corneal nerve fiber density (CNFD), branch density of corneal nerve fibers (CNBD), and corneal nerve fiber length (CNFL) [4]. Therefore, it might be possible to use these parameters from CCM to predict corneal complications such as epithelial defects after PPV.

In this study, we investigated the corneal nerves alteration associated with corneal complications after PPV. Our aim was to identify which parameter of corneal nerve plexus correlated with corneal complications after PPV. Additionally, receiver operating characteristic (ROC) curve was plotted and optimal cut-off values for predicting corneal complications following PPV was explored.

\section{Materials and Methods}

\section{Participants}

This study was performed under the approval from the institutional review board of He Eye Specialist Hospital (IRB(2020)K004.01) and conformed to the tenets of Declaration of Helsinki. All participants were informed of their rights and provided written informed consent before undergoing study procedures. Consecutive patients who underwent PPV in He Eye Specialist Hospital from April 1, 2020 to June 30, 2020 were enrolled in the study, including proliferative diabetic retinopathy (PDR), rhegmatogenous retinal detachment and vitreous hemorrhage form retinal vein occlusion and so on. Participants were excluded from the analysis if they had corneal surgery, corneal dystrophy, or cystic corneal disorders. Participants in this study were divided into two groups (group 1: with corneal complications after PPV and group 2: non corneal complications after PPV), these groups were named as follows: group $1=$ G1 and group $2=\mathrm{G} 2$.

The primary outcome measures in this study was corneal complications. Defined as corneal complications after PPV reported by Hiraoka et al. [5] and Chen et al. [1], as "the presence of corneal epithelial defects, corneal edema, or superficial punctate keratopathy lasting for at least 7 days after PPV". The definition of persistent corneal epithelial defects (PCED) as suggested by Tsubota et al. [6], is a corneal epithelial defect persisting for more than 2 weeks without improvement despite conventional treatment such as artificial tear drops or extended soft contact lens use.
All participants enrolled for this study were preoperatively assessed using a slit lamp biomicroscope, indirect ophthalmoscope and tonometer. Additionally, all participants underwent CCM assessment using a Heidelberg Retina Tomography III (Heidelberg Engineering, Heidelberg, Germany), which uses an additional lens (Rostock Cornea Module) for corneal observation. CCM was performed by an expert examiner (WH) who obtained images from the central cornea at the level of sub-basal nerve plexus (SNP). The assessor anaesthetized the eye prior to CCM assessment using one drop of $0.4 \%$ benoxinate hydrochloride (Santen Pharmaceutical, Osaka, Japan). To improve the adhesion between the cornea and applanation cap, Viscotears (Carbomer 980, 0.2\%; Novartis, North Ryde, UK) was used. Patients were asked to place their forehead against the headrest and fixate onto a light target in order for achieve centration. The objective lens of the microscope was gradually moved forward till gel came in contact with the cornea. The confocal scanning laser method allows a detailed visualization of the cornea and its layers separately within a $400-\mu \mathrm{m} \times 400-\mu \mathrm{m}$ viewing area. Five to eight images generated by Heidelberg Retina Tomography III provides an acceptable level of accuracy to quantify SNP morphology [7]. We chose and analyzed five images with high-clarity from the central SNP. Criteria for image selection were contrast, depth, and focus position.

\section{Analysis of corneal images}

Five high-clarity images (without motion artifact) of SNP were assessed for further quantitative evaluation. The assessment of SNP was based on the calculation of three parameters, which could be used to denote as a potential morphological state of nerve fiber $[8,9]$. These parameters included (1) CNFD: total number of nerve fibers observed within a frame (nerve fibers $/ \mathrm{mm}^{2}$ ), (2) CNBD: total number of nerve branches originating from major nerve trunks, per $\mathrm{mm}^{2}$ of corneal tissue (nerve branches $/ \mathrm{mm}^{2}$ ), and (3) CNFL: total length of nerve fibers and branches within a frame $\left(\mathrm{mm} / \mathrm{mm}^{2}\right)$ [8]. Analysis of the CCM images of SNP was carried out by Neuron J of Image J (National Institutes of Health, Bethesda, MD, USA). The average of measurements was used for further comparative analysis. All photographs were graded by two independent ophthalmol- 
ogists (HY and GQ) in a masked fashion.

\section{Surgical techniques}

All patients underwent an extensive 3-port PPV using 23-gauge instrumentation (OS4; Oertli, Berneck, Switzerland) and Resight 500 (Carl Zeiss Meditec, Dublin, CA, USA) for visualization under retrobulbar anesthesia. According to each subject's particular needs and requirements, fibrovascular membrane dissection were performed. Endolaser PRP was performed during the operation until all 4 quadrants had laser burns 1 burn length apart at least to the end of the midperipheral retina in PDR eyes. Vitreous replacement with air, long acting expansile gas mixtures (C2F6), fluid or 1000 centistoke silicone oil and subtenon's dexamethasone administration at the end of the surgery were left to the judgement of the surgeon. In case of a combined procedure, phacoemulsification was systematically performed before the PPV, through a $2.8-\mathrm{mm}$ clear corneal incision, with implantation of an acrylic foldable intraocular lens. Start time of the operation was recorded at the time of retrobulbar anesthesia, and the operation finish time was recorded following eyelid speculum removal at the end of the PPV. Postoperatively, patients were instructed to instill topical $1 \%$ prednisolone acetate, four times daily for 28 days, and topical $0.5 \%$ levofloxacin, four times daily for 7 days. Brinzolamide and timolol maleate eye drops would be topically given twice a day if intraocular pressure was $\geq 30 \mathrm{mmHg}$. Postoperative data collection was performed at five visits: day 1 , day 7 , day 14 , day 28 , and 3rd month.

\section{Statistical analysis}

Normality was assessed using histograms and Shapiro-Wilk test. Categoric variables were analyzed using chisquare test. Student $t$-test was used for comparison of groups with normally distributed variables, while Wilcoxon ranksum test for continuous non-normally distributed variables. All eyes were analyzed together in a multivariate logistic regression model to identify baseline factors that are predictive of corneal complications after PPV. ROC curve analysis was performed to identify the optimal cut-off point of the corneal fibers' parameters (at which sensitivity and specificity would be at a maximum) for the prediction of corneal complications after PPV. All statistical analyses were performed with IBM SPSS Statistics ver. 25.0 (IBM Corp., Armonk, NY, USA), alpha level (type I error) was set at 0.05 .

The inter-rater agreement for assessment of corneal fibers' parameters (CNFD, CNBD, and CNFL) was checked using intraclass coefficient (ICC). The agreement between reviewers was high: ICC $=0.986$ for CNFD evaluation, $\mathrm{ICC}=0.963$ for $\mathrm{CNBD}$, and $\mathrm{ICC}=0.983$ for CNFL assessment (all $p<0.001)$.

Table 1. Comparison of baseline characteristic among two groups

\begin{tabular}{lccc}
\hline & Group 1 $(\mathrm{n}=11)$ & Group 2 $(\mathrm{n}=83)$ & $p$-value \\
\hline Age (yr) & $55.00 \pm 9.66$ & $56.72 \pm 11.81$ & 0.645 \\
DM (yes : no) & $10: 1$ & $48: 35$ & 0.034 \\
Sex (male : female) & $5: 6$ & $44: 39$ & 0.642 \\
ECC (no/mm ${ }^{2}$ ) & $2,645.63 \pm 296.60$ & $2,628.80 \pm 354.14$ & 0.881 \\
Operative time (min) & $70.45 \pm 35.88$ & $57.41 \pm 19.53$ & 0.067 \\
Surgery procedure & $10: 1$ & $60: 23$ & 0.187 \\
(phacovitrectomy : single PPV) & $3: 8$ & & \\
Tamponade (silicone oil : gas) & & & 0.750 \\
Corneal fibers (mm/mm $\left.{ }^{2}\right)$ & $19.44 \pm 6.88$ & $26.84 \pm 7.53$ & \\
CNFL & $28.82 \pm 9.91$ & $37.10 \pm 10.16$ & 0.003 \\
CNFD & $55.84 \pm 21.08$ & $82.04 \pm 31.89$ & 0.013 \\
CNBD & & & 0.010 \\
\hline
\end{tabular}

Values are presented as mean \pm standard deviation or number.

$\mathrm{DM}=$ diabetes mellitus; $\mathrm{ECC}=$ endothelial cell count; $\mathrm{PPV}=$ pars plana vitrectomy; $\mathrm{CNFL}=$ corneal nerve fiber length; $\mathrm{CNFD}=$ corneal nerve fiber density; $\mathrm{CNBD}=$ branch density of corneal nerve fibers. 
Table 2. Corneal complications at 1 week after pars plana vitrectomy

\begin{tabular}{lccc}
\hline & $\begin{array}{c}\text { Diabetic } \\
\text { patients } \\
(\mathrm{n}=58)\end{array}$ & $\begin{array}{c}\text { Non-diabetic } \\
\text { patients } \\
(\mathrm{n}=36)\end{array}$ & $p$-value \\
\hline Group 1 $(\mathrm{n}=11)$ & $10(17.24)$ & $1(2.78)$ & 0.047 \\
Group 2 $(\mathrm{n}=83)$ & $48(82.76)$ & $35(97.22)$ & \\
\hline
\end{tabular}

Values are presented as number (\%).

Table 3. Multivariate logistic regression analysis of the associated risk factors with corneal complications following pars plana vitrectomy

\begin{tabular}{lccc}
\hline & $p$-value & Odds ratio & $95 \%$ CI \\
\hline Operative time & 0.140 & - & - \\
$\begin{array}{l}\text { Diabetes mellitus } \\
\text { Surgery }\end{array}$ & 0.431 & - & - \\
$\quad$ procedure & 0.154 & - & - \\
CNFL & 0.005 & 0.858 & $0.770-0.956$ \\
CNFD & 0.997 & - & - \\
CNBD & 0.934 & - & - \\
\hline
\end{tabular}

$\mathrm{CI}=$ confidence interval; $\mathrm{CNFL}=$ corneal nerve fiber length; $\mathrm{CNFD}=$ corneal nerve fiber density; $\mathrm{CNBD}=$ branch density of corneal nerve fibers.

\section{Results}

Ninety-four eyes of 94 patients were enrolled in this study, with 11 eyes with corneal complications after PPV in G1, and 83 eyes without corneal complications in G2. The mean age was $56.52 \pm 11.55$ years old, with a slight male predominance (52.12\%). The baseline characteristics of patients in both groups are presented in Table 1.

There was statistically significant difference between CNFL (19.44 \pm 6.88 vs. $26.84 \pm 7.53, p=0.003)$, CNFD (28.82 \pm 9.91 vs. $37.10 \pm 10.16, p=0.013)$, and CNBD (55.84 \pm 21.08 vs. $82.04 \pm 31.89, p=0.01)$ in two groups, respectively (Table 1).

Eleven eyes (11.70\%) developed corneal complications at 1 week after PPV in all patients. Ten eyes (10.6\%) with corneal complications were diabetic patients (Table 2), and eight (8.51\%) of them developed PCED on 2 weeks after PPV. PCED had been lasting for at least 3 months in four eyes $(4.26 \%)$, two eyes of them failed medicine therapy and progressed corneal ulcer, which eventually got amni-

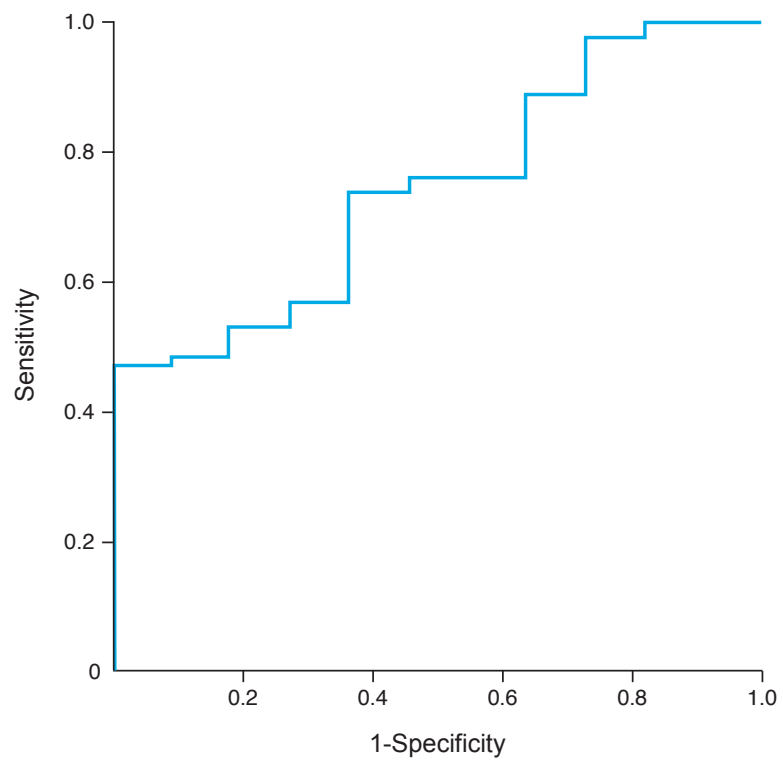

Fig. 1. Receiver operating characteristic curve analysis, the optimal cutoff value of corneal nerve fiber length in the prediction of corneal complications following pars plana vitrectomy. An area under the curve value is 0.743 , with $47 \%$ sensitivity and $100 \%$ specificity.

otic membrane transplantation and healed.

The variables of operative time, DM, three SNP parameters and operative procedure were enrolled in the multivariate logistic regression analysis. The result showed that CNFL was the only significant risk factor for corneal complications after PPV. Every micrometer decrease of CNFL increased the likelihood of achieving corneal complications after PPV by $17 \%$ (odds ratio, $0.858 ; 95 \%$ confidence interval, 0.770-0.956; $p=0.005$ ) (Table 3).

According to the ROC curve analysis, the optimal cutoff value of CNFL in the prediction of corneal complications following PPV was detected. The optimal cutoff value of CNFL to predict corneal complications following PPV was $<26.495 \mathrm{~mm} / \mathrm{mm}^{2}$, with an area under the curve value of $0.743,95 \%$ confidence interval $0.610-0.875$, sensitivity $47 \%$, and specificity $100 \%$ (Fig. 1).

\section{Discussion}

PPV allows the treatment for various vitreoretinal disorders involving the vitreous, retina and choroid, which may offer the opportunity for visual recovery. Corneal complications following PPV are comparatively common, with an 
incidence rate reaching up to $23.1 \%[1,3,10]$. In our study, the prevalence of corneal complications after PPV is $11.7 \%$, lower compared to previous studies. The technique of PPV is progressing very fast recently, including the updating of assisting lens used in the operation. Chiang et al. [3] reported that the lens they used was contact lens with lens ring fixed by scleral suture. Chen et al. [1] reported a handheld contact lens used with an assistant needed in their study and found a first-year resident assisting the operation was the risk factor for PCED. However, we used Resight 500 noncontact viewing system in this study, which does not need an assistant holding the lens or lens suture fixation, no eyes with corneal epithelium debridement, that could reduce the mechanical scratching to the cornea, this may be the reason to explain the low rate of corneal complication following PPV in our study, our result is also close to the rate $(15.9 \%)$ of corneal complications Al-Hinai et al. [10] reported, noncontact viewing system was used in his study too. Additional, Chiang et al. [3] reported that operative time was a risk factor for corneal epithelial defect, the operative time in his study was nearly two hours, but the mean operative time was just $58.94 \pm 22.19$ minutes in our study. Though there is no significant difference between operative time in G1 and G2, G1 has the tendency of more time used compared to $\mathrm{G} 2(70.45 \pm 35.88$ vs. $57.41 \pm$ 19.53 minutes, $p=0.067$ ), so less operative time used might be another reason to support our result. The rate of PCED is $8.51 \%$ in our study, slightly higher compared to previous studies, Chen et al. [1] reported that the rate of PCED was $4.6 \%$ in their study, they also found DM was a risk factor for PCED. PDR patients were predominant $(61.7 \%)$ in our study, and all the PCED patients were with PDR, that could explain that.

The cornea is densely innervated with nerves, containing $\mathrm{A}-\delta$ and unmyelinated $\mathrm{C}$ fibers derived from the ophthalmic division of the trigeminal nerve [11,12]. In addition to their sensory and reflex functions, corneal nerves also have significant trophic effects on the cornea and play an important role in the preservation of ocular surface health through the stimulation of corneal wound healing after corneal injuries [13]. Several studies have been conducted to investigate the exact mechanism by which the corneal nerve fibers maintain a healthy cornea and promote wound healing after corneal injuries. The results of these studies suggest that there is a trophic relationship between corneal nerves and epithelial cells. Corneal nerves can stimulate epithelial cell growth, proliferation, differentiation and type IV collagen formation through the release of neurotrophins and neuropeptides [14]. In our study, CNFL, CNFD and CNBD is significant decreased in the G1 compared to G2, respectively. Decreased corner nerve fibers might influence the corneal wound healing.

Diabetic keratopathy is characterized by abnormal innervation of the cornea that results in decreased sensitivity and impaired epithelial wound healing [15]. SNP is frequently affected in diabetic patients with neuropathy and the changes depend on the severity of neuropathy. It has been found that the number of fibers, number of beadings, and branching pattern of fibers in the sub-basal plexus decreases in cases of mild to severe neuropathy $[9,16,17]$. These are primarily caused by changes within the arrangement of the corneal epithelial basement membrane, deposition of glycation products, corneal nerve endings damage and reduction in tear secretion, that leads to oxidative stress during the hyperglycemic condition [18]. Several studies had testified DM was a risk factor for corneal complications after PPV [1,3], though more diabetic patients had corneal complications after PPV in this study, DM was not noted as the risk factor in the multivariate logistic regression analysis. CNFL was the only significant risk factor for corneal complications after PPV.

The corneal complications after PPV would rarely happen if CNFL $\geq 26.495 \mathrm{~mm} / \mathrm{mm}^{2}$ according to the ROC analysis result, the occurrence of corneal complications after PPV might be about $50 \%$ if CNFL $<26.495 \mathrm{~mm} / \mathrm{mm}^{2}$. These results could help the retinal surgeons to evaluate the risk factors of corneal complications following PPV before the operation. Interestingly, Scarr et al. [19] also suggested CNFL be classified as abnormal for the NPDR and PDR groups adhering to the cutoff values.

The limitations of this study are the relatively small study population and single center study. However, to the best of our knowledge, this is the first study to use preoperative corneal nerve parameters to predict the corneal complications following PPV. This study is also void of any bias from pharmaceutical sponsorship; hence, the data are generalizable to the typical retina practice patient.

In conclusion, preoperative corneal nerve parameters (CNFL, CNFD, and CNBD) decreases in PPV patients with postoperative corneal complications, the rate of corneal complications following PPV may decrease with the noncontact viewing system, the decrease of CNFL may 
predict corneal complications following PPV. Regular preoperative CCM test in PPV patients could be considered.

\section{Conflict of Interest}

No potential conflict of interest relevant to this article was reported.

\section{References}

1. Chen HF, Yeung L, Yang KJ, Sun CC. Persistent corneal epithelial defect after pars plana vitrectomy. Retina 2016;36:148-55.

2. Chen WL, Lin CT, Ko PS, et al. In vivo confocal microscopic findings of corneal wound healing after corneal epithelial debridement in diabetic vitrectomy. Ophthalmology 2009;116:1038-47.

3. Chiang WY, Lee JJ, Kuo HK, et al. Factors associated with corneal epithelial defects after pars plana vitrectomy. Int Ophthalmol 2018;38:105-110.

4. Kalteniece A, Ferdousi M, Adam S, et al. Corneal confocal microscopy is a rapid reproducible ophthalmic technique for quantifying corneal nerve abnormalities. PLoS One 2017;12:e0183040.

5. Hiraoka M, Amano S, Oshika T, et al. Factors contributing to corneal complications after vitrectomy in diabetic patients. Jpn J Ophthalmol 2001;45:492-5.

6. Tsubota K, Goto E, Shimmura S, Shimazaki J. Treatment of persistent corneal epithelial defect by autologous serum application. Ophthalmology 1999;106:1984-9.

7. Vagenas D, Pritchard N, Edwards K, et al. Optimal image sample size for corneal nerve morphometry. Optom $\mathrm{Vis} \mathrm{Sci}$ 2012;89:812-7.

8. Kallinikos P, Berhanu M, O’Donnell C, et al. Corneal nerve tortuosity in diabetic patients with neuropathy. Invest Oph- thalmol Vis Sci 2004;45:418-22.

9. Malik RA, Kallinikos P, Abbott CA, et al. Corneal confocal microscopy: a non-invasive surrogate of nerve fibre damage and repair in diabetic patients. Diabetologia 2003;46:683-8.

10. Al-Hinai AS. Corneal epithelial defect after pars plana vitrectomy. Oman J Ophthalmol 2017;10:162-6.

11. Al-Aqaba MA, Alomar T, Miri A, et al. Ex vivo confocal microscopy of human corneal nerves. Br J Ophthalmol 2010;94:1251-7.

12. Nielsen NV, Lund FS. Diabetic polyneuropathy: corneal sensitivity, vibratory perception and Achilles tendon reflex in diabetics. Acta Neurol Scand 1979;59:15-22.

13. Al-Aqaba MA, Dhillon VK, Mohammed I, et al. Corneal nerves in health and disease. Prog Retin Eye Res 2019;73: 100762 .

14. Baker KS, Anderson SC, Romanowski EG, et al. Trigeminal ganglion neurons affect corneal epithelial phenotype: influence on type VII collagen expression in vitro. Invest Ophthalmol Vis Sci 1993;34:137-44.

15. Bikbova G, Oshitari T, Baba T, et al. Diabetic corneal neuropathy: clinical perspectives. Clin Ophthalmol 2018;12:9817.

16. Hossain P, Sachdev A, Malik RA. Early detection of diabetic peripheral neuropathy with corneal confocal microscopy. Lancet 2005;366:1340-3.

17. Midena E, Brugin E, Ghirlando A, et al. Corneal diabetic neuropathy: a confocal microscopy study. J Refract Surg 2006;22(9 Suppl):S1047-52.

18. Priyadarsini S, Whelchel A, Nicholas S, et al. Diabetic keratopathy: insights and challenges. Surv Ophthalmol 2020;65:513-29.

19. Scarr D, Lovblom LE, Ostrovski I, et al. Agreement between automated and manual quantification of corneal nerve fiber length: implications for diabetic neuropathy research. J Diabetes Complications 2017;31:1066-73. 